\title{
Corrigendum: The Mouse Intestinal Bacterial Collection (miBC) provides host-specific insight into cultured diversity and functional potential of the gut microbiota
}

Ilias Lagkouvardos, Rüdiger Pukall, Birte Abt, Bärbel U. Foesel, Jan P. Meier-Kolthoff, Neeraj Kumar, Anne Bresciani, Inés Martínez, Sarah Just, Caroline Ziegler, Sandrine Brugiroux, Debora Garzetti, Mareike Wenning, Thi P. N. Bui, Jun Wang, Floor Hugenholtz, Caroline M. Plugge, Daniel A. Peterson, Mathias W. Hornef, John F. Baines, Hauke Smidt, Jens Walter, Karsten Kristiansen, Henrik B. Nielsen, Dirk Haller, Jörg Overmann, Bärbel Stecher and Thomas Clavel

Nature Microbiology 1, 16131 (2016); published 8 August 2016; corrected 17 October 2016

In the original version of this paper, several of the bacterial genus and species names were incorrect or incompatible with formal taxonomic validation and have had to be modified. The relevant names and descriptions have been amended in all versions of the Article. In addition, Supplementary Figs 3,4 and Supplementary Tables 1,4,5,11 have been replaced. 